\title{
Poor response to artesunate treatment in two patients with severe malaria on the Thai- Myanmar border
}

\author{
Aung Pyae Phyo ${ }^{1,6^{*}} \mathbb{D}$, Kyaw Kyaw Win ${ }^{5}$, Aung Myint Thu' ${ }^{1}$, Lei Lei Swe ${ }^{1}$, Htike Htike ${ }^{5}$, Candy Beau ${ }^{1}$, \\ Kanlaya Sriprawat ${ }^{1}$, Markus Winterberg², Stephane Proux', Mallika Imwong ${ }^{3}$, Elizabeth A. Ashley 2,4,6 \\ and Francois Nosten ${ }^{1,4}$
}

\begin{abstract}
Background: Malaria has declined dramatically along the Thai-Myanmar border in recent years due to malaria control and elimination programmes. However, at the same time, artemisinin resistance has spread, raising concerns about the efficacy of parenteral artesunate for the treatment of severe malaria.

Case presentation: In November 2015 and April 2017, two patients were treated for severe malaria with parenteral artesunate. Quinine was added within $24 \mathrm{~h}$ due to an initial poor response to treatment. The first patient died within $24 \mathrm{~h}$ of starting treatment and the second did not clear his peripheral parasitaemia until 11 days later. Genotyping revealed artemisinin resistance Kelch-13 markers.
\end{abstract}

Conclusions: Reliable efficacy of artesunate for the treatment of severe malaria may no longer be assured in areas where artemisinin resistance has emerged. Empirical addition of parenteral quinine to artesunate for treatment is recommended as a precautionary measure.

Keywords: Severe malaria, Artemisinin resistance, Quinine, Artesunate, Kelch 13

\section{Background}

Parenteral artesunate and quinine are the recommended first-line drugs for the treatment of severe Plasmodium falciparum malaria [1]. Artesunate has a broader stagespecificity of action than quinine [2] and as a result acts more rapidly, i.e., artesunate kills circulating ring-stage parasites, which are later cleared by the spleen, whereas quinine does not. Evidence from multi-centre clinical trials of patients with severe malaria in Southeast Asia [3] and Africa [4] have shown a significant mortality reduction following artesunate treatment compared to quinine in adults and children.

However, recent emergence of artemisinin resistance characterized by a slow parasite clearance is raising the

\footnotetext{
*Correspondence: aungpyaephyo@shoklo-unit.com

${ }^{1}$ Shoklo Malaria Research Unit, Mahidol-Oxford Tropical Medicine Research Unit, Faculty of Tropical Medicine, Mahidol University, PO Box 46, Mae Sot, Tak 63110, Thailand

Full list of author information is available at the end of the article
}

possibility of erosion of the life-saving properties of the artemisinin derivatives when treating patients with severe malaria, although it has not been possible to study this under trial conditions due to the low number of cases. There were only four cases of severe malaria hospitalized in Shoklo Malaria Research Unit between 2015 to date compared to 38 cases in 2011 [5]. Here, two cases of severe malaria with a poor response to parenteral artesunate are presented.

\section{Case presentations \\ Case 1: At the village}

A 30-year-old male farmer with no past history of malaria presented to a malaria post close to the Myanmar-Thai border in November 2015, with 3 days of fever and headache. He showed no signs of severity and was found to be positive for $P$. falciparum with an HRP-2-based rapid diagnostic test. He was treated with oral artemetherlumefantrine by the village malaria post (first dose 
supervised). The next morning, he returned complaining of anuria, when the village malaria worker referred him to a clinic in Thailand.

\section{At the clinic}

On arrival, the patient appeared slightly jaundiced, was apyrexial and fully conscious. The blood smear revealed a high P. falciparum asexual parasitaemia of $20 \%$ of infected red blood cells (iRBC) or 757,368 $\mu \mathrm{L}$. The presence of schizonts $(352 \mu \mathrm{L})$ and gametocytes $(1216 \mu \mathrm{L})$ was noted, as well as malaria pigment in the neutrophils. Capillary blood haematocrit (Hct) was $30 \%$, blood glucose $82 \mathrm{mg} \%$, pulse 88 beats/min, respiratory rate $32 / \mathrm{min}, \mathrm{SPO}_{2} 98 \%$ in air, blood pressure $110 / 60 \mathrm{mmHg}$. His liver was palpable, $3 \mathrm{~cm}$ below the costal margin and his spleen was not palpable. Physical examination was otherwise unremarkable. The patient was treated with intravenous artesunate $(2.4 \mathrm{mg} / \mathrm{kg})$ because of the oligo-anuria and high parasitaemia [6] and rehydrated with $2.5 \mathrm{~L}$ of normal saline over $6 \mathrm{~h}$. His vital signs, blood glucose, conscious level and parasitaemia were monitored 4-hourly and these remained within normal limits.
However, the patient remained oligo-anuric and was referred to another clinic equipped with a biochemistry analyser. Blood urea nitrogen [BUN] (normal range) was $91.8(2.5-7.1 \mathrm{mmol} / \mathrm{L})$, creatinine $2.5(0.67-1.17 \mathrm{mg} /$ $\mathrm{dL})$, potassium $4.47(3.5-5.1 \mathrm{mmol} / \mathrm{L})$, sodium 135.8 (136-145 mmol/L), bicarbonate 4.9 (23-29 mmol/L), blood glucose was $74 \mathrm{mg} / \mathrm{dL}$ and Hct dropped to $22 \%$, so $350 \mathrm{~mL}$ whole blood was transfused. His Glasgow Coma Score (GCS) was 15/15; he was eating and walking unsupported, had a respiratory rate of $40 / \mathrm{min}$, clear lungs and a temperature of $36.9^{\circ} \mathrm{C}$, blood pressure of $90 / 40 \mathrm{mmHg}$. His asexual parasitaemia was now $27 \%$ of iRBC or $746,064 \mu \mathrm{L}$ (> $80 \%$ of early ring stage), gametocytaemia $1824 \mu \mathrm{L}$, schizontaemia $32 \mu \mathrm{L}$. A loading dose $(20 \mathrm{mg} / \mathrm{kg})$ of quinine 4-hourly infusion was added. Four hours after the start of the loading dose quinine infusion (quinine in $500 \mathrm{~mL}$ of dextrose 10\%) the patient's condition was unchanged and his asexual parasitaemia was now $26 \%$ iRBC or $718,432 \mu \mathrm{L}$ (> 80\% early ring stage), gametocytaemia $1920 \mu \mathrm{L}$, schizontaemia $16 \mu \mathrm{L}$ (Fig. 1), blood glucose was $104 \mathrm{mg} / \mathrm{dL}$. A second dose of intravenous artesunate $2.4 \mathrm{mg} / \mathrm{kg}$ was given. After $4.5 \mathrm{~L}$ of fluid (including fluid bolus) the patient passed $60 \mathrm{~mL}$ of

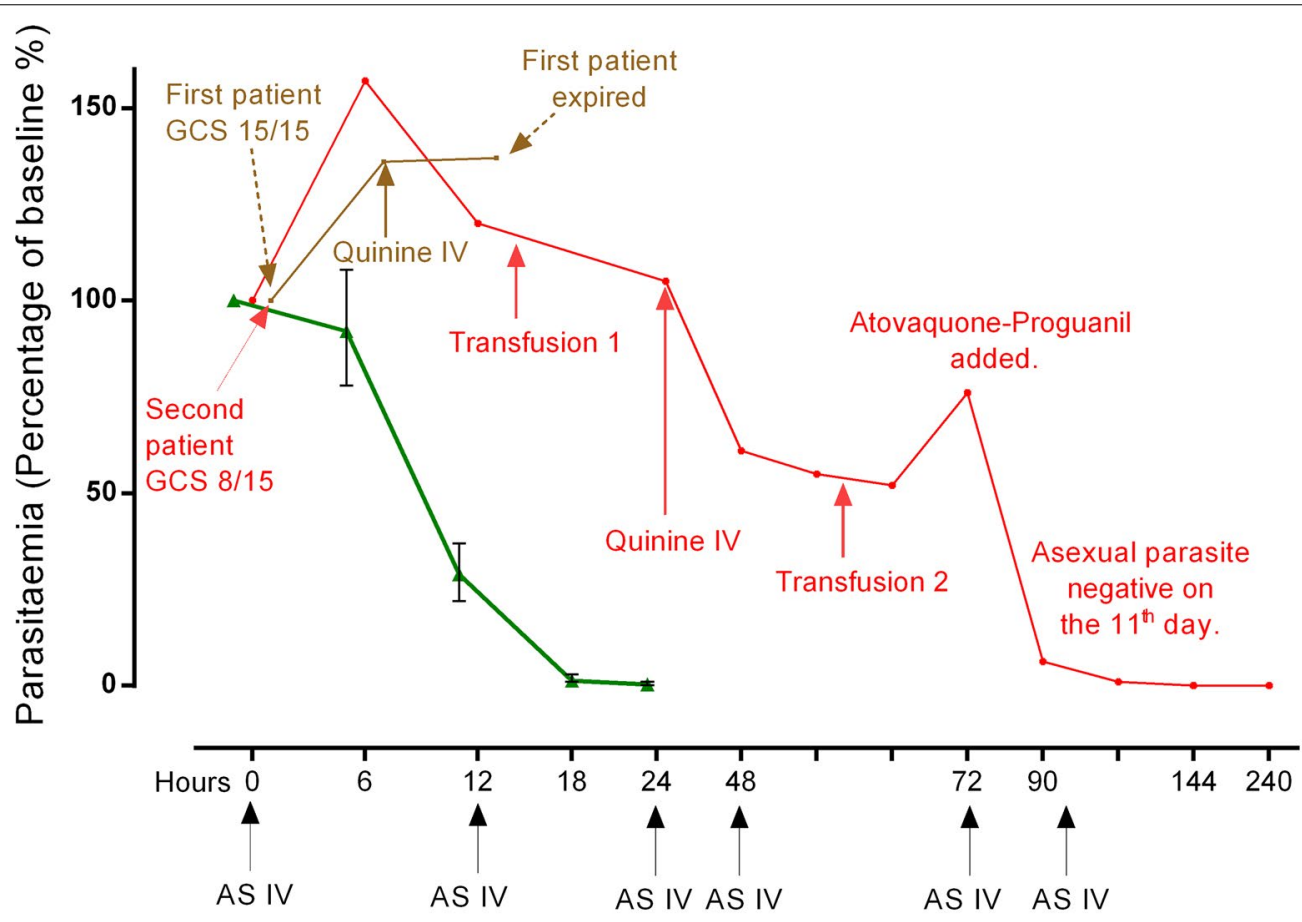

Uncomplicated hyperparasitaemia patients

treated with oral artesunate before the emergence of artemisinin resistance $2004^{*}$ (median;IQR)

$\rightarrow$ Parasite Clearance of the first patient who died. $\rightarrow$ Parasite Clearance of the second patient.

Fig. 1 Clinical and parasitological response of two severe malaria cases compared to those patients with uncomplicated hyperparasitaemia who received artesunate per oral (green) (median: IQR) 
high-coloured urine and his temperature rose to $39.7^{\circ} \mathrm{C}$. Fourteen hours after arrival, the patient became unconscious, had a generalized seizure followed by cardiac arrest. Resuscitation was unsuccessful.

\section{Case 2}

On 3 April, 2017, an 18 years old male was admitted with cerebral malaria: $P$. falciparum asexual parasitaemia $38 \%$ iRBC or $1,050,016 \mu \mathrm{L}$ (late stage trophozoite $40 \%)$. HIV rapid test was positive, which was confirmed subsequently with a baseline CD4 count of 39 cells/ $\mathrm{mL}$. The patient's GCS was $8 / 15$, tympanic temperature $39.0{ }^{\circ} \mathrm{C}$, blood glucose $51 \mathrm{mg} / \mathrm{dL}$, and blood pressure $120 / 80 \mathrm{mmHg}$. His liver was palpable, $3 \mathrm{~cm}$ below the costal margin and his spleen was not palpable, although confirmed to be present on abdominal ultrasonography. Intravenous artesunate $(2.4 \mathrm{mg} / \mathrm{kg})$ was given on admission, after $12 \mathrm{~h}$ and again after $24 \mathrm{~h}$ as per protocol [1], i.e., three doses within the first $24 \mathrm{~h}$. However, $24 \mathrm{~h}$ after the start of treatment, the parasitaemia was still $34 \%$ of iRBC or $1,069,107 \mu \mathrm{L}$ (> 90\% late trophozoites) and a loading dose of quinine $(20 \mathrm{mg} / \mathrm{kg})$ was added. At hour 24 , he received a blood transfusion of $350 \mathrm{~mL}$ whole blood.

After $72 \mathrm{~h}$, the patient recovered consciousness and started eating. However, the parasitaemia had increased again (Fig. 1). Atovaquone-proguanil combination was given in addition to the intravenous artesunate and quinine. By hour-90, the asexual parasitaemia was $7 \%$ iRBC or $200,221 \mu \mathrm{L}$ and he received a second transfusion. Intravenous artesunate and quinine infusion were given for 7 days in total. By day 10 his malaria slide was negative. The patient was discharged on day 12 of hospital admission.

\section{Anti-malarial drug quality}

The anti-malarials used were artemether-lumefantrine (Macleods Pharmaceuticals: EAB 5412B) and artesunate injection (Atlantic pharma: LA170333) provided by the Global Fund malaria programme. Quinine injection (A.N.B laboratories Co. Ltd: 555009) and atovaquoneproguanil (Malarone GSK: GS 0009 Dec 2018) were purchased from the supplier. All the drugs were in date.

\section{Laboratory tests}

All blood slides were double-checked by an expert microscopist of Shoklo Malaria Research Unit.

\section{Parasite genotyping}

Leftover blood from the patients was sent for parasite Kelch-13 genotyping with patient or relative written consent. Both had kelch mutant parasites: patient 1 with the P441L mutation and patient 2 the C580Y mutation.
Microsatellite genotyping of flanking regions of C580Y haplotype of the Kelch-13 gene revealed a different C580Y lineage from those described recently in Western Cambodia/Northeastern Thailand/Southern Laos/Myanmar, suggesting a different parental origin [7].

\section{Plasma drug concentrations}

In Case 1, artesunate or DHA were not detected which was not unexpected since the blood sampling time was $\sim 24 \mathrm{~h}$ after the second (last) dose of artemetherlumefantrine. However, lumefantrine was also undetectable, which could be due to poor absorption of drug since the tablets were taken with water [8] or non-adherence since the second dose was not supervised.

In Case 2, the plasma concentration of artesunate after $\sim 2 \mathrm{~h}$ of intravenous injection was $105 \mathrm{ng} / \mathrm{mL}$ and DHA was $1530 \mathrm{ng} / \mathrm{mL}$, which were well above the plasma levels providing maximum parasiticidal effect [9].

\section{In vitro parasite culture}

Parasite isolates taken from patient 2, after two doses of intravenous artesunate injection ( $\sim 2 \mathrm{~h}$ of second dose) grew normally in drug free plate (Additional file 1).

\section{In vitro sensitivity assay}

The in vitro Ring-stage Survival Assay ( $\mathrm{RSA}^{0-3 h}$ ) was performed on the parasites obtained from these two patients (Fig. 2). In both cases in vitro assay confirmed that these isolates were resistant to artemisinin (see Methods in Additional file 2).

\section{Conclusions}

Intravenous artesunate is the treatment of choice for severe malaria globally [3]. However, in this report, the parasitaemias in two patients with severe malaria treated with intravenous artesunate did not decline as expected and one patient died. Despite treatment, a proportion of patients will always die as a result of severe malaria. The presence of schizonts and neutrophils with malaria pigment [6] on the peripheral blood smear suggest that a significant proportion of the total parasite biomass was already undergoing sequestration. Artesunate and quinine may not have been able to reach these sequestered parasites. Similarly, the very high parasitaemia with slow parasite clearance in the second patient may have been partly attributable to his HIV status, since the development of opsonizing antibodies to variant surface antigens [10] and phagocytosis of opsonized infected erythrocytes by macrophages are impaired by HIV infection [11, 12]. Indeed, the time taken to clear the parasitaemia was far longer than usual following quinine monotherapy of presumed artemisinin-sensitive infections (parasite clearance in 83-96 h) $[13,14]$. Nevertheless, there is 


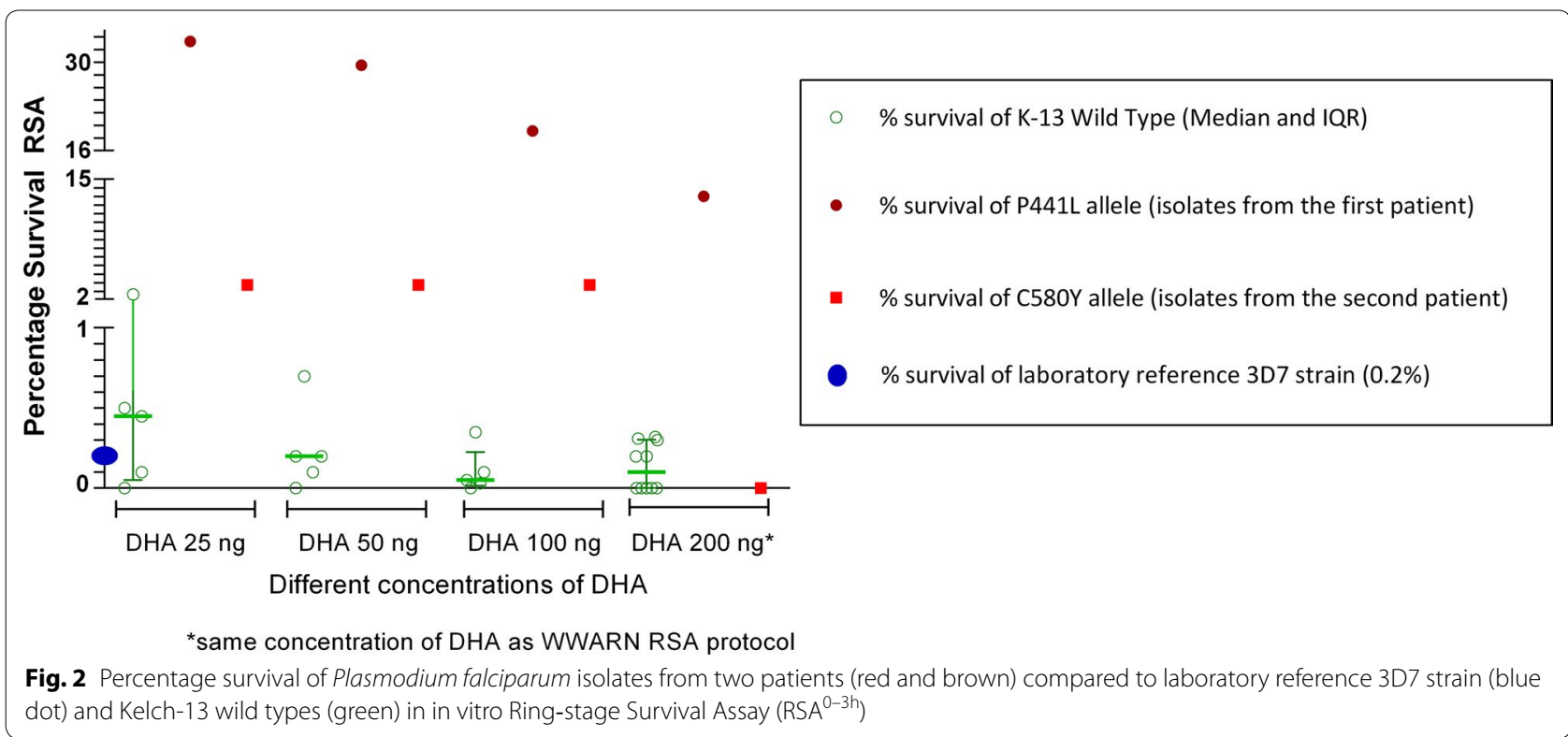

uncertainty and clinicians treating patients with severe falciparum malaria acquired in areas where resistance to artemisinin is documented are confronted with difficult decisions when the parasite clearance fails to follow the expected decline.

The unique advantage of the artemisinins that makes them superior to other anti-malarials in treating severe malaria is their broad stage-specificity of action (especially on the early rings). This advantage is severely compromised by artemisinin resistance with implications for the treatment of severe malaria. A previous study of 69 patients conducted in 1998 [15] showed no improvement in parasite clearance from combining parenteral artesunate and quinine; however the safety of this combination was acceptable $[15,16]$. The absence of an additive effect of artesunate and quinine at that time was explained by the broad-spectrum parasiticidal activity of artesunate, which results in very fast in vivo parasite clearance. This property is severely compromised in artemisinin-resistant infections.

In the absence of conclusive evidence, to minimize the risk to individual patients, it is recommended that all patients treated for severe malaria acquired in areas where artemisinin resistance is established should receive immediate empirical parenteral artesunate and quinine with frequent monitoring of parasite density.

\section{Additional files}

Additional file 1. Parasite isolates collected after two doses of parenteral artesunate growing in drug-free plate.

Additional file 2. Methods and other table.

\section{Authors' contributions}

APP, LLS, CB, KKW, HH, and EA were responsible for the treatment of the patients. SP confirmed the blood smear findings. APP and AMT collected the detailed information from the village. Ml did the parasite genotyping, KS did the in vitro RSA assay, MW analysed the drug levels. APP, EA and FN coordinated and wrote up the manuscript. All authors read and approved the final manuscript.

\section{Author details}

${ }^{1}$ Shoklo Malaria Research Unit, Mahidol-Oxford Tropical Medicine Research Unit, Faculty of Tropical Medicine, Mahidol University, PO Box 46, Mae Sot, Tak 63110, Thailand. ${ }^{2}$ Mahidol-Oxford Tropical Medicine Research Unit, Faculty of Tropical Medicine, Mahidol University, Bangkok, Thailand. ${ }^{3}$ Department of Molecular Tropical Medicine and Genetics, Faculty of Tropical Medicine, Mahidol University, Bangkok, Thailand. ${ }^{4}$ Centre for Tropical Medicine and Global Health, Nuffield Department of Medicine, University of Oxford, Oxford, UK. ${ }^{5}$ Mae Tao Clinic, 702, Moo 1, Tha Sai Luad, Mae Sot, Tak 63110,

Thailand. ${ }^{6}$ Myanmar Oxford Clinical Research Unit, Yangon, Myanmar.

\section{Acknowledgements}

The staff of Mae Tao clinic and Shoklo Malaria Research Unit are thanked for their work in managing these two patients.

\section{Competing interests}

The authors declare that they have no competing interests.

\section{Availability of data and materials}

The datasets used and/or analysed during the current study are available from the corresponding or last author on reasonable request.

\section{Consent for publication}

Written informed consent to report these cases was obtained from the parents of the first patient and directly from the second patient after recovery.

\section{Ethics approval and consent to participate} Not applicable.

\section{Funding}

Funding for the Shoklo Malaria Research Unit came from the Wellcome Trust, UK. The funders had no role in study design, data collection and analysis, decision to publish, or preparation of the manuscript. 


\section{Publisher's Note}

Springer Nature remains neutral with regard to jurisdictional claims in published maps and institutional affiliations.

Received: 22 November 2017 Accepted: 10 January 2018

Published online: 15 January 2018

\section{References}

1. World Health Organization. Guidelines for the treatment of malaria. Geneva: World Health Organization; 2015.

2. White NJ. Assessment of the pharmacodynamic properties of antimalarial drugs in vivo. Antimicrob Agents Chemother. 1997;41:1413-22.

3. Dondorp A, Nosten F, Stepniewska K, Day N, White N, South East Asian Quinine Artesunate Malaria Trial Group. Artesunate versus quinine for treatment of severe falciparum malaria: a randomised trial. Lancet. 2005;366:717-25.

4. Dondorp AM, Fanello Cl, Hendriksen IC, Gomes E, Seni A, Chhaganlal KD et al. Artesunate versus quinine in the treatment of severe falciparum malaria in African children (AQUAMAT): an open-label, randomised trial. Lancet. 2010;376:1647-57.

5. Carrara VI, Lwin KM, Phyo AP, Ashley E, Wiladphaingern J, Sriprawat K, et al. Malaria burden and artemisinin resistance in the mobile and migrant population on the Thai-Myanmar border, 1999-2011: an observational study. PLoS Med. 2013;10:e1001398.

6. Phu NH, Day N, Diep PT, Ferguson DJP, White NJ. Intraleucocytic malaria pigment and prognosis in severe malaria. Trans R Soc Trop Med Hyg. 1995;89:200-4.

7. Imwong M, Suwannasin K, Kunasol C, Sutawong K, Mayxay M, Rekol H, et al. The spread of artemisinin-resistant Plasmodium falciparum in the Greater Mekong subregion: a molecular epidemiology observational study. Lancet Infect Dis. 2017;17:491-7.
8. Ashley EA, Stepniewska K, Lindegardh N, Annerberg A, Kham A, Brockman $A$, et al. How much fat is necessary to optimize lumefantrine oral bioavailability? Trop Med Int Health. 2007;12:195-200.

9. Angus BJ, Thaiaporn I, Chanthapadith K, Suputtamongkol Y, White NJ. Oral artesunate dose-response relationship in acute falciparum malaria. Antimicrob Agents Chemother. 2002;46:778-82.

10. Keen J, Serghides L, Ayi K, Patel SN, Ayisi J, van Eijk A, et al. HIV impairs opsonic phagocytic clearance of pregnancy-associated malaria parasites. PLoS Med. 2007:4:e181.

11. Ludlow LE, Zhou J, Tippett E, Cheng W-J, Hasang W, Rogerson SJ, et al. HIV-1 inhibits phagocytosis and inflammatory cytokine responses of human monocyte-derived macrophages to P. falciparum infected erythrocytes. PLoS ONE. 2012;7:e32102.

12. Joice R, Frantzreb C, Pradham A, Seydel KB, Kamiza S, Wirth DF, et al. Evidence for spleen dysfunction in malaria-HIV co-infection in a subset of pediatric patients. Mod Pathol. 2016;29:381-90.

13. Eder M, Farne H, Cargill T, Abbara A, Davidson RN. Intravenous artesunate versus intravenous quinine in the treatment of severe falciparum malaria: a retrospective evaluation from a UK centre. Pathog Glob Health. 2012;06:181-7.

14. Kurth F, Develoux M, Mechain M, Clerinx J, Antinori S, Gjørup IE, et al. Intravenous artesunate reduces parasite clearance time, duration of intensive care, and hospital treatment in patients with severe malaria in Europe: the TropNet severe malaria study. Clin Infect Dis. 2015;61:1441-4.

15. Newton PN, Chierakul W, Ruangveerayuth R, Silamut K, Teerapong P, Krudsood S, et al. A comparison of artesunate alone with combined artesunate and quinine in the parenteral treatment of acute falciparum malaria. Trans R Soc Trop Med Hyg. 2001;95:519-23.

16. Bartoloni A, Tomasoni L, Bartalesi F, Galli L, Sani S, Veloci S, et al. Combined intravenous treatment with artesunate and quinine for severe malaria in Italy. Am J Trop Med Hyg. 2010;83:274-6.

\section{Submit your next manuscript to BioMed Central and we will help you at every step:}

- We accept pre-submission inquiries

- Our selector tool helps you to find the most relevant journal

- We provide round the clock customer support

- Convenient online submission

- Thorough peer review

- Inclusion in PubMed and all major indexing services

- Maximum visibility for your research

Submit your manuscript at www.biomedcentral.com/submit
O Biomed Central 